\title{
A study on equilibrium and kinetics of ion exchange of alkaline earth metals using an inorganic cation exchanger - zirconium titanium phosphate
}

\author{
AMIN JIGNASA, THAKKAR RAKESH and CHUDASAMA UMA* \\ Applied Chemistry Department, Faculty of Technology and Engineering, MS University of Baroda, \\ Vadodara 390001 \\ e-mail: uvcres@yahoo.com
}

MS received 25 August 2005; revised 18 January 2006

\begin{abstract}
An advanced inorganic cation exchange material of the class of tetravalent metal acid (TMA) salt, zirconium titanium phosphate (ZTP), has been synthesized by a modified sol-gel technique. ZTP has been characterized by elemental analysis (ICP-AES), thermal analysis (TGA), FTIR and X-ray diffraction studies. The Nernst-Planck equation has been used to study the forward and reverse ion exchange kinetics of $\mathrm{Mg}$ (II), $\mathrm{Ca}$ (II), $\mathrm{Sr}$ (II) and $\mathrm{Ba}$ (II) with $\mathrm{H}$ (I) at four different temperatures. The mechanism of exchange is particle diffusion, as confirmed by the linear $\tau$ (dimensionless time parameter) vs $t$ (time) plots. The exchange process is thus controlled by the diffusion within the exchanger particles for the systems studied herein. Further, various kinetic parameters like self-diffusion coefficient $\left(D_{0}\right)$, energy of activation $\left(E_{a}\right)$ and entropy of activation $\left(\Delta S^{*}\right)$ have been evaluated under conditions favouring a particle diffusion-controlled mechanism.
\end{abstract}

Keywords. Kinetics of ion exchange; zirconium titanium phosphate; cation exchanger; tetravalent metal acid salts; inorganic ion exchanger.

\section{Introduction}

Tetravalent metal acid (TMA) salts are cation exchangers, obtainable in both amorphous and crystalline forms, and possessing the general formula $\mathrm{M}(\mathrm{IV})\left(\mathrm{HXO}_{4}\right)_{2} \cdot n \mathrm{H}_{2} \mathrm{O}$, where $\mathrm{M}(\mathrm{IV})=\mathrm{Zr}, \mathrm{Ti}, \mathrm{Sn}, \mathrm{Ce}$, Th etc. and $\mathrm{X}=\mathrm{P}, \mathrm{W}, \mathrm{As}, \mathrm{Mo}, \mathrm{Sb}$ etc. These materials possess structural hydroxyl groups, the $\mathrm{H}$ of the $-\mathrm{OH}$ being the exchangeable sites. A number of cations can be exchanged with $\mathrm{H}^{+}$due to which the material possesses cation-exchange properties. Depending on the stoichiometry of the reagents used, temperature at which they are mixed, rate of addition, mode of mixing, $\mathrm{pH}$ etc. the resultant materials vary in water content, composition and crystallinity. These materials show great promise in preparative reproducibility, ion-exchange behaviour and stability, both thermal and chemical. TMA salts have multiphase applications. Owing to their granular nature and producibility in a range of mesh sizes, and selectivity for certain metal ions, these materials have

\footnotetext{
*For correspondence
}

occupied a prominent position in the field of separation science.

There is currently high interest in engineering mixed materials leading to the formation of new solid-state structures and materials with new composite properties. Mixed materials are novel multifunctional substances that offer a wide range of interesting properties. New mixed materials of the class of TMA salts, i.e. compounds containing two different cations and a given anion or two different anions and a cation, are interesting since they show improved ion exchange properties and selectivity for particular metal ions in comparison to their single salt counterparts. A literature survey shows that mixed materials containing two anions and a cation have been widely investigated. However, not much work has been done on mixed materials containing two cations and an anion.

Zirconium and titanium phosphates are now very well-known inorganic ion exchange materials of the class of TMA salts. Although these compounds are not very different as exchangers, slight differences do exist owing to the difference in their ion size para- 
meters. It was thought that if $\mathrm{Ti}^{4+}$ is incorporated in the matrix of zirconium phosphate or visa versa, the ion exchange properties of mixed zirconium-titanium phosphate could change considerably as compared to the single salt counterparts.

Kinetics of exchange enables us to understand the viability of an ion exchange material in separation technology. A literature survey shows that extensive work has been carried out on kinetics of ion exchange using TMA single salts (containing a cation and an anion) as well as mixed materials (containing two anions and a cation). ${ }^{1}$ It was therefore thought of interest to study the kinetics of ion exchange on mixed materials containing two cations and an anion, which has not been much explored.

In the present endeavour, an advanced inorganic material of the class of tetravalent metal acid (TMA) salt, zirconium titanium phosphate (ZTP), has been synthesized by a modified sol-gel technique. The material has been characterized by elemental analysis (ICP-AES), thermal analysis (TGA), FTIR and Xray diffraction studies. Forward and reverse ion exchange kinetics of $\mathrm{Mg}$ (II), Ca (II), Sr (II) and $\mathrm{Ba}$ (II) with $\mathrm{H}$ (I) have been studied at various temperatures. A mechanism of exchange has been proposed and various kinetic parameters like self-diffusion coefficient $\left(D_{0}\right)$, energy of activation $\left(E_{a}\right)$ and entropy of activation $\left(\Delta S^{*}\right)$ have been evaluated.

\section{Experimental}

All chemicals and reagents used are of analytical grade.

\subsection{Synthesis of ZTP}

A solution containing $0.1 \mathrm{M} \mathrm{TiCl} \mathrm{Tin}_{4}$ and $0.1 \mathrm{M}$ $\mathrm{ZrOCl}_{2} \cdot 8 \mathrm{H}_{2} \mathrm{O}$ in $10 \% \mathrm{w} / \mathrm{v} \mathrm{H}_{2} \mathrm{SO}_{4}(100 \mathrm{ml})$ was prepared. To the above solution $0.2 \mathrm{M}(200 \mathrm{ml})$ $\mathrm{NaH}_{2} \mathrm{PO}_{4} \cdot 2 \mathrm{H}_{2} \mathrm{O}$ was added drop wise (flow rate =

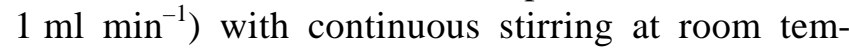
perature. On complete precipitation the gel obtained was stirred for a further $5 \mathrm{~h}$. It was then kept in contact with mother liquor overnight, filtered, washed with conductivity water for removal of adhering ions (chloride and sulphate) and dried at room temperature. The material was then broken down to the desired particle size [30-60 mesh (ASTM)] by grinding and sieving. Five grams of this material was taken and treated with $50 \mathrm{ml}$ of $1 \mathrm{M} \mathrm{HNO}_{3}$ for 30 min with occasional shaking. The sample was then separated from the acid by decantation and treated with conductivity water for removal of adhering acid. This process (acid treatment) was repeated at least five times. After final washing, the material was dried at room temperature.

\subsection{Ion exchange capacity (IEC)}

Ion exchange capacity $\left(\mathrm{Na}^{+}\right.$exchange $)$was determined by column method. ${ }^{2}$ The column was prepared in a burette, provided with glass wool at the bottom. It was filled half way with distilled water, preventing air traps and then $0.5 \mathrm{~g}$ of the ion exchanger was accurately weighed and transferred through a dry funnel. The water inside the column was kept at a level of about $1 \mathrm{~cm}$ above the material. A $250 \mathrm{ml}$ solution of sodium acetate was added into the column and elution was carried out at a flow rate of $0.5 \mathrm{ml} \mathrm{min}^{-1}$. The eluant was collected in a $500 \mathrm{ml}$ conical flask and then titrated against $0 \cdot 1 \mathrm{~N}$ $\mathrm{NaOH}$ solution. The IEC of the exchanger in milli equivalent per gram $\left(\mathrm{meq} \mathrm{gm}^{-1}\right.$ ) is given by, $a v / w$ where $a$ is the molarity of the $\mathrm{NaOH}$ solution, $v$ is the volume of $\mathrm{NaOH}$ required for titration and $w$ is the weight of the exchanger.

\subsection{Instrumentation}

ZTP has been analyzed for zirconium, titanium and phosphorus by ICP-AES. Thermal analysis was performed on a Shimadzu DT-30 thermal analyzer at a heating rate of $10 \mathrm{~K}$ per minute. FTIR spectra was obtained using $\mathrm{KBr}$ wafer on a Perkin-Elmer paragon 1000 spectrophotometer. $X$-ray diffractograms $(2 \theta=$ $5-90^{\circ}$ ) were obtained on an X-ray diffractometer Brucker AXS D8 using $\mathrm{Cu}-\mathrm{K}_{\alpha}$ radiation with a nickel filter. For kinetic studies, a shaker with a temperature variation of $\pm 0.5 \mathrm{~K}$ was used.

\subsection{Kinetic studies}

2.4a Concentration variation study: Exchanger particles of definite mesh size [30-60 mesh (ASTM), mean radii $\sim 178 \eta \mathrm{m}]$ of $\mathrm{H}^{+}$form was used to evaluate various kinetic parameters. The concentration effect on the mechanism of exchange was studied at $303 \mathrm{~K}$ by taking different metal ion concentrations from 0.002 to $0.012 \mathrm{M}$ at intervals of $0.002 \mathrm{M}$ for the forward process. In the reverse process, the concentration effect on the mechanism of exchange was studied at $303 \mathrm{~K}$ by taking different hydrogen ion 
concentrations from 0.002 to $0.020 \mathrm{M}$ at intervals of $0.002 \mathrm{M}$.

\section{4a Temperature variation study: Fractions} $(20 \mathrm{ml}$ each) of $0.006 \mathrm{M}$ metal ion solutions prepared in DMW were shaken with $200 \mathrm{mg}$ of exchanger in several stoppered conical flasks at the desired temperatures $(303,313,323,333 \mathrm{~K})$ for different time intervals $(0 \cdot 5,1 \cdot 0,2 \cdot 0,3 \cdot 0,4 \cdot 0 \mathrm{~min})$. The supernatant liquid was removed after every prescribed time interval and the metal ion concentration evaluated by EDTA titration. Each set was repeated twice and mean values were taken for calculations. In the reverse process, the exchanger was taken in the metal form and was shaken with $20 \mathrm{ml}$ of $0.020 \mathrm{M}$ $\mathrm{HNO}_{3}$. The supernatant liquid in this case was titrated against a standard $\mathrm{NaOH}$ solution to determine the amount of $\mathrm{H}^{+}$ions exchanged on the exchanger.

\section{Results and discussion}

\subsection{Characterization}

ZTP synthesized is obtained as white hard granules. ICP-AES shows the ratio of $\mathrm{Zr}: \mathrm{Ti}: \mathrm{P}$ to be $1: 1: 2$. The $\mathrm{Na}^{+}$ion exchange capacity of ZTP at room temperature is $3.36 \mathrm{meq} \mathrm{gm}^{-1}$.

The FTIR spectrum of ZTP exhibits a broad band in the region $\sim 3400.0 \mathrm{~cm}^{-1}$, which is attributed to asymmetric and symmetric hydroxy - $\mathrm{OH}$ stretches. A sharp medium band at $\sim 1620 \mathrm{~cm}^{-1}$ is attributed to aquo $(\mathrm{H}-\mathrm{O}-\mathrm{H})$ bending. A band in the region $\sim 1031.8 \mathrm{~cm}^{-1}$ is attributed to $\mathrm{P}=\mathrm{O}$ stretching.

TGA of ZTP shows a sharp change within the temperature range of $353 \mathrm{~K}$ to $453 \mathrm{~K}$ corresponding to the loss of external water molecules, after which a gradual weight loss is observed till $793 \mathrm{~K}$. This may be due to the condensation of structural hydroxyl groups.

X-ray diffractogram of ZTP does not exhibit any sharp peaks indicating that ZTP is an amorphous material.

\subsection{Kinetic studies}

It is very well established that the rate-determining step in an ion exchange process is the interdiffusion of the counter ions. ${ }^{3}$ The rate-controlling mechanism can be film diffusion, if the slow step is diffusion across the hydrodynamic film that surrounds the exchanger particles or particle diffusion, if the slow step is diffusion inside the exchanger beads themselves. In the first case, a concentration gradient is set up within the liquid film, whereas inside the exchanger a uniform concentration of ion, prevails. In the second case the concentration gradient occurs within the exchanger, while the film has a uniform composition. High metal ion concentration, relatively large particle size of the exchanger and vigorous shaking of the exchanging mixture favour a particle diffusion-controlled process. ${ }^{4}$

Kinetics of ion exchange can be explained with respect to fractional attainment of equilibrium $U(t)$ for a particle diffusion-controlled phenomenon by $U(t)=1-\left(Q_{A}(t) / Q_{\mathrm{A}}^{0}\right)=\left[C_{0}-C(t) / C_{0}-C_{\propto}\right]^{5}, \quad$ where, $Q_{A}(t)$ and $Q_{\mathrm{A}}^{0}$ are the amount of $\mathrm{A}$ at time $\mathrm{t}$ and initial amount of $\mathrm{A}$ in the ion exchanger respectively (A being the counter ion), while $C(t), C_{\propto}$ and $C_{0}$ are metal ion concentration at time $(t)$, equilibrium metal ion concentration and initial metal ion concentration respectively. $U(t)$ is seen to be dependant only on the magnitude of the dimensionless time parameter $\tau$. According to the explicit approximation, ${ }^{6-8}$ $U(t)$ for a particle diffusion controlled phenomenon is given by, $U(\tau)=\left\{1-\exp \left[\pi^{2}\left(f_{1}(\alpha) \tau+f_{2}(\alpha) \tau^{2}+\right.\right.\right.$ $\left.\left.\left.f_{3}(\alpha) \tau^{3}\right)\right]\right\}^{1 / 2}$, where, $\tau=D_{A} t / r_{0}^{2}$ and $\alpha$ (mobility ratio) $=D_{A} / D_{B} . D_{A}$ and $D_{B}$ are diffusion coefficients of counter ions $A$ and $B$ respectively in the ion exchanger phase, $r_{0}=$ bead radius and $t=$ time. The plots of $\tau$ vs $t$ for different metal ion concentrations are plotted. The concentration at which the plot of $\tau$ vs $t$ is a straight line passing through the origin, confirms a particle diffusion-controlled exchange. ${ }^{5}$ This concentration is thus the minimum concentration for particle diffusion-controlled exchange.

The concentration effect on the mechanism of ion exchange was studied at $303 \mathrm{~K}$ by taking different metal ion concentrations and the plots of $\tau$ vs $t$ were plotted. For the forward process, plots of $\tau$ vs $t$ at metal ion concentrations $\geq 0.006 \mathrm{M}$ are straight lines passing through the origin, which confirms a particle diffusion-controlled exchange at these concentrations. ${ }^{5}$ In the reverse process, however, this limit is $\geq 0.020 \mathrm{M}$. This difference in the concentration limits for the forward and reverse process may be due to the different degrees of ionization of an acid and its salt. In the first case the exchanger behaves as an acid because of its $\mathrm{H}^{+}$form, while in the second case it behaves as a salt due to its metal form. For further kinetic studies, this minimum concentration $(0.006 \mathrm{M}$ for forward process and $0.020 \mathrm{M}$ for backward process) is taken because at this concentration the Don- 
Table 1. Values of various parameters for forward and reverse exchanges of $\mathrm{M}^{2+}-\mathrm{H}^{+}$.

\begin{tabular}{|c|c|c|c|c|c|c|c|c|c|}
\hline \multirow{2}{*}{$\begin{array}{l}\text { Migrat- } \\
\text { ing } \\
\text { ion }\end{array}$} & \multirow{2}{*}{$\begin{array}{c}\text { Ion } \\
\text { present } \\
\text { in the } \\
\text { exchanger }\end{array}$} & \multicolumn{4}{|c|}{$\begin{array}{l}\text { Equilibrium values }\left(\mathrm{meq} \mathrm{gm}^{-1}\right) \\
\quad\left(\text { slope }(S) \times 10^{5}\left(\mathrm{~s}^{-1}\right)\right) \text { at }\end{array}$} & \multirow{2}{*}{$\begin{array}{c}\text { Ionic } \\
\text { radii of } \\
\text { migrating } \\
\text { ion }(\AA)\end{array}$} & \multirow{2}{*}{$\begin{array}{c}D_{0} \\
\left(\mathrm{~m}^{2} \mathrm{~s}^{-1}\right)\end{array}$} & \multirow{2}{*}{$\begin{array}{c}E_{a} \\
\left(\mathrm{~kJ} \mathrm{~mol}^{-1}\right)\end{array}$} & \multirow{2}{*}{$\begin{array}{c}\Delta S^{*} \\
\left.\mathrm{~kJ} \mathrm{~mol}^{-1}\right)\end{array}$} \\
\hline & & $303 \mathrm{~K}$ & $313 \mathrm{~K}$ & $323 \mathrm{~K}$ & $333 \mathrm{~K}$ & & & & \\
\hline$\overline{M g}(\mathrm{II})$ & $\mathrm{H}(\mathrm{I})$ & $0 \cdot 34($ & $0 \cdot 44(7 \cdot 17)$ & $0.45(8 \cdot 00)$ & $0 \cdot 46$ & 0.65 & $2.26 \times 10^{-10}$ & 11.96 & $-81 \cdot 04$ \\
\hline $\mathrm{H}(\mathrm{I})$ & $\operatorname{Mg}(\mathrm{II})$ & $0.93(1.17)$ & $1.22(1.50)$ & $1.28(1.67)$ & $1 \cdot 32(2 \cdot 00)$ & $0 \cdot 29$ & $1.68 \times 10^{-10}$ & $15 \cdot 34$ & $-83 \cdot 52$ \\
\hline $\mathrm{Ca}(\mathrm{II})$ & $\mathrm{H}(\mathrm{I})$ & $0.44(5 \cdot 33)$ & $0.46(6.50)$ & $0.47(7.67)$ & $0 \cdot 49(8 \cdot 17)$ & 0.99 & $2.22 \times 10^{-10}$ & $12 \cdot 17$ & $-81 \cdot 19$ \\
\hline $\mathrm{H}(\mathrm{I})$ & $\mathrm{Ca}(\mathrm{II})$ & $1.52(0.67)$ & $1.62(0.83)$ & $1.68(1 \cdot 16)$ & $1.72(1.50)$ & 0.29 & $3.16 \times 10^{-8}$ & $30 \cdot 62$ & $-39 \cdot 96$ \\
\hline $\operatorname{Sr}(\mathrm{II})$ & $\mathrm{H}(\mathrm{I})$ & $0.43(4.33)$ & $0.44(6.00)$ & $0.46(7.50)$ & $0.47(8.83)$ & $1 \cdot 13$ & $3.80 \times 10^{-9}$ & $19 \cdot 89$ & $-57 \cdot 59$ \\
\hline $\mathrm{H}(\mathrm{I})$ & $\operatorname{Sr}(\mathrm{II})$ & $1.45(0.50)$ & $1.52(0.83)$ & $1.59(1 \cdot 17)$ & $1.62(1.50)$ & 0.29 & $3.16 \times 10^{-8}$ & $30 \cdot 62$ & $-39 \cdot 96$ \\
\hline $\mathrm{Ba}(\mathrm{II})$ & $\mathrm{H}(\mathrm{I})$ & $0.31(7.00)$ & $0.33(8.50)$ & $0.35(11.50)$ & $0.36(16.67)$ & 1.35 & $4.27 \times 10^{-9}$ & $19 \cdot 09$ & $-56 \cdot 61$ \\
\hline $\mathrm{H}(\mathrm{I})$ & $\mathrm{Ba}(\mathrm{II})$ & $1 \cdot 17(0 \cdot 50)$ & $1.22(0.67)$ & $1 \cdot 34(1 \cdot 17)$ & $1.44(1.33)$ & $0 \cdot 29$ & $1.88 \times 10^{-8}$ & $29 \cdot 47$ & $-44 \cdot 30$ \\
\hline
\end{tabular}

nan exclusion is predominant, which prevents the interference of co-ion in the exchange process. ${ }^{4}$

Effect of temperature on ion exchange was studied at $303,313,323,333 \mathrm{~K}$ by taking different metals $\left(\mathrm{Mg}^{2+}, \mathrm{Ca}^{2+}, \mathrm{Sr}^{2+}\right.$ and $\left.\mathrm{Ba}^{2+}\right)$. Equilibrium values at different temperatures for forward and reverse exchanges for $\mathrm{M}^{2+}-\mathrm{H}^{+}$are shown in table 1 . It shows that equilibrium values increase with temperature. This may be due to the fact that mobility of the ion increases with an increase in temperature. Further, equilibrium values for the forward exchange are lower as compared to reverse exchange. This may be due to the fact that at higher concentrations, more exchange takes place. In the forward process the metal ion concentration is $0.006 \mathrm{M}$, while in the reverse process the hydrogen ion concentration is $0.020 \mathrm{M}$.

From kinetic study experiments, the plots of $\tau$ vs $t$ at four different temperatures for metal (II)-hydrogen (I) and hydrogen (I)-metal (II) exchanges are obtained. These plots are straight lines passing through the origin, confirming a particle diffusioncontrolled phenomenon for forward and reverse processes at metal and hydrogen ion concentrations of 0.006 $\mathrm{M}$ and 0.020 $\mathrm{M}$ respectively.

Slopes $(S)$ obtained from various $\tau$ vs $t$ plots for all the metal ions are given in table 1 . Values of $D_{A}$ are calculated using the relation, $D_{A}=S \times r_{0}^{2}$, and $\ln D_{A}$ plotted against $1 / T$. These plots are straight lines verifying the validity of the Arrhenius relation, $D_{A}=D_{0} \exp \left(-E_{a} / R T\right)$. The energy of activation $\left(E_{a}\right)$ and the pre-exponential constant $\left(D_{0}\right)$ are calculated from these plots. The entropy of activation $\left(\Delta S^{*}\right)$ is then calculated ${ }^{9}$ using $D_{0}$ in the equation, $D_{0}=2.72$ $d^{2} k T / h \exp \left(\Delta S^{*} / R\right)$ where, $d=$ average distance between two exchanging sites ${ }^{9}$ taken as $5 \AA$ and other terms have their usual meaning.

Values of the self-diffusion co-efficient $\left(D_{0}\right)$, energy of activation $\left(E_{a}\right)$ and entropy of activation $\left(\Delta S^{*}\right)$ thus obtained are summarized in table 1.
From table 1 it is observed that the self-diffusion coefficient is highest for $\mathrm{Ba}^{2+}$. This could be explained as being due to the fact that the extent of hydration of an ion in aqueous solution depends upon the size and charge of the ion. Size of $\mathrm{Ba}^{2+}$ being the highest, the $\mathrm{Ba}^{2+}$ ion is least hydrated and therefore its self-diffusion co-efficient, is highest. According to the values of self-diffusion coefficients, the selectivity order is $\mathrm{Ba}^{2+}>\mathrm{Sr}^{2+}>\mathrm{Mg}^{2+}>\mathrm{Ca}^{2+}$. Further, it is observed that $E a$ values are low for the forward reaction as compared to the reverse reaction, indicative of ease in the forward exchange process. In general, $\Delta S^{*}$ values are observed to be more negative for the forward than the reverse process. This is probably due to the lower degree of ionization of a salt than the corresponding acid and hence strong affinity of the exchanger for the metal ions. In other words, more negative $\Delta S^{*}$ values for the forward process than the reverse process, indicate the presence of more active exchangeable sites in the exchanger and strong preference for metal ions. This is also in keeping with $E_{a}$ values. Such observations have been made earlier by other workers. ${ }^{10}$

\section{Conclusion}

The ZTP synthesized exhibits good ion exchange capacity, thermal and chemical resistitivity. The forward and reverse ion exchange kinetics using alkaline earth metals reveal the mechanism of exchange to be particle diffusion as confirmed by the linear $\tau$ (dimensionless time parameter) vs $t$ (time) plots. Further, various kinetic parameters like selfdiffusion coefficient $\left(D_{0}\right)$, Energy of activation $\left(E_{a}\right)$ and entropies of activation $\left(\Delta S^{*}\right)$ have been evaluated under condition favouring a particle diffusioncontrolled mechanism. The above studies thus reveal the promising use of ZTP as a cation exchanger. 
Further, study on the effects of different electrolyte concentrations and $\mathrm{pH}$ values on distribution coefficient $\left(K_{d}\right)$ is in progress.

\section{References}

1. Varshney K G, Agrawal S and Varshney K 1984 Colloids Surfaces 9189

2. Nabi S A and Rao R K 1981 J. Indian Chem. Soc. 11 1030

3. Schulze G 1915 Z. Phys. Chem. 89168
4. Helfferich F 1962 Ion exchange (New York: McGraw-Hill) pp. 255, 282

5. Barrer R M 1941 Diffusion in and through solids (New York: Cambridge University Press) pp. 29, 50

6. Helfferich F and Plesset M S 1958 J. Chem. Phys. 28418

7. Plesset M S, Helfferich F and Franklin J N $1958 \mathrm{~J}$. Chem. Phys. 291064

8. Varshney K G, Varshney K and Agrawal S 1986 Colloids and Surfaces 1867

9. Barrer R M, Bartholomew R F and Rees L V C 1961 Phys. Chem. Solids 2112

10. Varshney K G, Gupta U and Maheshwari S M 1997 React. Kinet. Catal. Lett. 61127 